\title{
Relativistic coupled-cluster single-double method applied to alkali-metal atoms
}

\author{
Rupsi Pal and M. S. Safronova \\ Department of Physics and Astronomy, University of Delaware, Newark, DE 19716-2570, USA \\ W. R. Johnson \\ University of Notre Dame, Notre Dame, Indiana 46556, USA \\ Andrei Derevianko \\ University of Nevada, Reno, NV 89557-0042, USA \\ Sergey G. Porsev \\ Petersburg Nuclear Physics Institute, Leningrad district, Gatchina, \\ 188300, Russia and University of Nevada, Reno, NV 89557-0042, USA
}

\begin{abstract}
A relativistic version of the coupled-cluster single-double (CCSD) method is developed for atoms with a single valence electron. In earlier work, a linearized version of the CCSD method (with extensions to include a dominant class of triple excitations) led to accurate predictions for energies, transition amplitudes, hyperfine constants, and other properties of monovalent atoms. Further progress in high-precision atomic structure calculations for heavy atoms calls for improvement of the linearized coupled-cluster methodology. In the present work, equations for the single and double excitation coefficients of the Dirac-Fock wave function, including all non-linear coupled-cluster terms that contribute at the single-double level are worked out. Contributions of the non-linear terms to energies, electric-dipole matrix elements, and hyperfine constants of low-lying states in alkali-metal atoms from $\mathrm{Li}$ to $\mathrm{Cs}$ are evaluated and the results are compared with other calculations and with precise experiments.
\end{abstract}

PACS numbers: 31.15.Dv 32.10.-f 32.10.Fn 32.10.Hq 32.70.Cs

\section{INTRODUCTION}

A relativistic version of the coupled-cluster singledouble (CCSD) approximation for monovalent atoms is developed. In this approximation, single and double excitations of the (frozen-core) Dirac-Fock wave function for an atom with one valence electron are included to all orders in perturbation theory. The relativistic CCSD is an extension of an earlier relativistic single-double (SD) approximation, in which all nonlinear coupled-cluster terms were omitted. The relativistic SD approximation provides a method closely related to the configurationinteraction method for including classes of correlation corrections to Dirac-Fock wave functions to all-orders in perturbation theory. When modified to include the dominant triple excitations, the SD method led to accurate predictions for energies, transition matrix elements, lifetimes, hyperfine constants, and polarizabilities in alkalimetal atoms (see, for example, Refs. 1, 2, 3, 4, 5, 6]). Owing to recent improvements in the precision of atomic experiments, it is important to go beyond the relativistic SD approximation and include the non-linear coupledcluster terms.

The coupled-cluster method is an all-order extension of many-body perturbation theory introduced into nuclear physics by Coester and Kümmel [7, 8] and adapted to atomic and molecular physics by Cižek and Paldus [9, 10, 11]. A comprehensive review of the coupledcluster method and its applications in quantum chemistry is given in Ref. [12]. Relativistic CCSD calcula- tions for monovalent atoms have been carried out previously by Ynnerman et al. 13] for transition energies in Li-like U, by Eliav et al. 14] for ionization and excitation energies of alkali-metal atoms, by Lim et al. [15] for polarizabilities of alkali-metal atoms from Li to element 119, by Mårtensson-Pendrill [16] for charge radii of francium isotopes, by Landau et al. 17] for electron affinities in alkali-metal atoms $\mathrm{Na}$ to element 119, by Chaudhuri et al. [18 for ionization and excitation energies in $\mathrm{Rb}$ and Cs, by Porsev and Derevianko 19] for properties of Na, by Derevianko and Porsev [20] for properties of Cs, and by Sahoo et al. [21] for parity nonconservation in $\mathrm{Cs}$ and $\mathrm{Ba}^{+}$. Among the nonrelativistic applications of the CCSD method to alkali-metal atoms, we mention those of Lindgren 22] who calculated energies and hyperfine constants of $2 s$ and $2 p$ states in $\mathrm{Li}$ and Salomonson and Ynnerman [23] who evaluated energies and hyperfine constants of $3 s$ and $3 p$ states in Na.

The relativistic SD method for alkali-metal atoms was introduced in Ref. 24], where it was used to obtain energy levels, fine-structure intervals, hyperfine constants, and electric-dipole matrix elements in $\mathrm{Li}$ and $\mathrm{Be}^{+}$. Later, the SD method was applied successfully to study properties of heavier alkali-metal atoms and monovalent ions [1, 2, 3, 4, 5, 6]. Despite the success of the SD allorder method (and its extension to include important triples) in predicting many properties of monovalent systems, there are various instances where it fails to produce accurate results. For example, the magnetic-dipole hyperfine constant for the $7 d_{5 / 2}$ state of Fr calculated using 
the SD method significantly disagrees with the experimental value [25]. The $n d_{5 / 2}$ hyperfine constants in $\mathrm{Cs}$ provide other such examples [26]. This issue is not limited to the hyperfine constants; correlation corrections to electric-dipole $6 d_{j}-6 p_{j^{\prime}}$ matrix elements in $\mathrm{Rb}$ are so large that they change the sign of the matrix elements [4]. The large differences between third-order and all-order values noted in Ref. [4] lead to the conclusion that the omitted higher-order contributions are significant. Cases where correlation corrections are extremely large lead to poor accuracy for the SD method, which is generally of high-precision; in such cases, a more accurate treatment of correlations is mandatory.

Another motivation for further development of the relativistic SD method is the study of parity nonconservation (PNC) in heavy atoms. One goal of PNC studies is to provide atomic-physics tests of the standard model of the electroweak interactions through determination of the weak charge $Q_{W}$; another is to extract nuclear anapole moments from PNC measurements. A precise calculation of the PNC amplitude (together with an uncertainty estimate of the calculation) is necessary to derive a value of the weak charge $Q_{W}$ from experimental measurements. The accuracy of the most advanced experimental study of PNC (which was carried out in Cs) is $0.3 \%$ 27], while the accuracy of the corresponding theoretical calculations is about $0.5 \%$ (see [28] and references therein). The difference between the value of $Q_{W}$ extracted from experiment and the value inferred from the standard model stands at $1 \sigma[20]$. More accurate experiments, coupled with improvements in the calculation of PNC amplitude will lead to a more accurate determination of $Q_{W}$ and a more stringent test of the standard model.

Recently, a rather complete treatment of a class of triple excitations that included the valence electron and two core electrons was carried out in Refs. [19, 20] for $\mathrm{Na}$ and $\mathrm{Cs}$, respectively. Contributions of quadratic valence non-linear terms were also calculated and found to be relatively large. As a result, further development of the all-order method must include a complete treatment of the non-linear terms at the SD level.

In this work, we include all valence and core non-linear coupled-cluster corrections to the SD equations, leading to a complete set of CCSD equations, and we study their effects on various atomic properties of alkali-metal atoms. In particular, we calculate ionization energies and hyperfine constants for the ground $n s$ states and the $n p_{1 / 2}$, $n p_{3 / 2}$ excited states of $\mathrm{Li}, \mathrm{Na}, \mathrm{K}, \mathrm{Rb}$, and $\mathrm{Cs}$. Reduced electric-dipole matrix elements for the $n s-n p_{1 / 2}$ and $n s-n p_{3 / 2}$ transitions are also calculated. We give a detailed breakdown of various non-linear contributions in order to identify the most important effects and to measure the influence of core non-linear terms. Comparison of our calculations with other theoretical calculations and with experimental results is presented.

\section{COUPLED-CLUSTER METHOD}

In coupled-cluster theory, the wave function of an atom with one valence electron in a state $v$ is written as

$$
\left|\Psi_{v}\right\rangle=\exp (S)\left|\Phi_{v}\right\rangle
$$

where $\left|\Phi_{v}\right\rangle$ is the lowest-order atomic wave function for atomic state $v$, which taken to be a frozen core DiracFock (DF) wave function, and where the wave operator (the operator that maps the DF wave function onto the exact wave function) is expressed in exponential form $\exp (S)$. The exponential function in Eq. (1) can be expanded to give

$$
\left|\Psi_{v}\right\rangle=\left(1+S+\frac{1}{2} S^{2}+\cdots\right)\left|\Phi_{v}\right\rangle .
$$

The cluster operator $S$ is expressed as a sum of $n$-particle excitations $S_{n}$ of the lowest-order wave function

$$
S=S_{1}+S_{2}+\cdots .
$$

As the number of excitations increases, the complexity of the wave function increases. The computational complexity rises dramatically beyond the double excitation term $S_{2}$. Retaining only single and double excitations, Eq. (2) may be written

$$
\begin{aligned}
\left|\Psi_{v}\right\rangle= & \left(1+S_{1}+S_{2}+\frac{1}{2} S_{1}^{2}+S_{1} S_{2}+\frac{1}{6} S_{1}^{3}\right. \\
& \left.+\frac{1}{2} S_{2}^{2}+\frac{1}{2} S_{1}^{2} S_{2}+\frac{1}{24} S_{1}^{4}+\cdots\right)\left|\Phi_{v}\right\rangle .
\end{aligned}
$$

The one-electron excitation $S_{1}$ may be either an excitation of a core electron or an excitation of the valence electron. Correspondingly, the single core and valence excitations are given by

$$
\begin{aligned}
S_{1 c} & =\sum_{m a} \rho_{m a} a_{m}^{\dagger} a_{a}, \\
S_{1 v} & =\sum_{m \neq v} \rho_{m v} a_{m}^{\dagger} a_{v} .
\end{aligned}
$$

Similarly, for double core and valence excitations

$$
\begin{aligned}
S_{2 c} & =\frac{1}{2} \sum_{m n a b} \rho_{m n a b} a_{m}^{\dagger} a_{n}^{\dagger} a_{b} a_{a}, \\
S_{2 v} & =\sum_{m n b} \rho_{m n v b} a_{m}^{\dagger} a_{n}^{\dagger} a_{b} a_{v} .
\end{aligned}
$$

The expansion coefficients $\rho_{m a}$ and $\rho_{m v}$ are referred to later as single core and valence excitation coefficients, while $\rho_{m n a b}$ and $\rho_{m n v a}$ are referred to as double core and valence excitation coefficients, respectively. In Eqs. (5) and (6), $a_{i}^{\dagger}$ and $a_{i}$ are creation and annihilation operators for an electron state $i$. Here and in subsequent formulas, the indices from the beginning of the alphabet $a, b, \ldots$ designate core states, indices from the middle of the alphabet $m, n, \ldots$ designate excited states, the index $v$ 
labels the valence state, and indices $i, j, k$ and $l$ designate arbitrary states.

In the SD method, only terms linear in the excitation coefficients are retained, and all remaining terms are omitted. Substituting Eqs. (5) and (6) into Eq. (4) and retaining the terms linear in the excitation coefficients yields the single-double (SD) all-order wave function

$$
\begin{aligned}
\left|\Psi_{v}\right\rangle= & {\left[1+\sum_{m a} \rho_{m a} a_{m}^{\dagger} a_{a}+\frac{1}{2} \sum_{m n a b} \rho_{m n a b} a_{m}^{\dagger} a_{n}^{\dagger} a_{b} a_{a}\right.} \\
& \left.+\sum_{m \neq v} \rho_{m v} a_{m}^{\dagger} a_{v}+\sum_{m n b} \rho_{m n v b} a_{m}^{\dagger} a_{n}^{\dagger} a_{b} a_{v}\right]\left|\Phi_{v}\right\rangle .
\end{aligned}
$$

To derive the equations for the excitation coefficients (see Ref. 24] for details), the SD all-order wave function (7) is substituted into the many-body Schrödinger equation

$$
H\left|\Psi_{v}\right\rangle=E\left|\Psi_{v}\right\rangle
$$

where the Hamiltonian $H$ is the relativistic no-pair Hamiltonian [29], which can be written in secondquantized form as $H=H_{0}+V$, where

$$
\begin{aligned}
H_{0} & =\sum_{i} \epsilon_{i}\left[a_{i}^{\dagger} a_{i}\right] \\
V & =\frac{1}{2} \sum_{i j k l} g_{i j k l}\left[a_{i}^{\dagger} a_{j}^{\dagger} a_{l} a_{k}\right] \\
& +\sum_{i j}\left(V_{\mathrm{DF}}-U\right)_{i j}\left[a_{i}^{\dagger} a_{j}\right]+\frac{1}{2} \sum_{a}\left(V_{\mathrm{DF}}-2 U\right)_{a a} .
\end{aligned}
$$

In the no-pair Hamiltonian, contributions from negativeenergy (positron) states are omitted. Products of operators enclosed in brackets, such as $\left[a_{i}^{\dagger} a_{j}^{\dagger} a_{l} a_{k}\right]$, designate normal products with respect to a closed core. The quantity $U$ in Eq. (10) is the model potential used in the Dirac equation defining single-particle orbitals. In this work, $U_{i j}$ is taken to be frozen-core Dirac-Fock potential

$$
U_{i j}=\left(V_{\mathrm{DF}}\right)_{i j}=\sum_{a}\left(g_{i a j a}-g_{i a a j}\right) .
$$

Such a choice of the potential significantly simplifies calculation since the second term in Eq. (10) disappears. The quantities $g_{i j k l}$ are two-body Coulomb matrix elements:

$$
g_{i j k l}=\int d^{3} r \int d^{3} r^{\prime} \psi_{i}^{\dagger}(\mathbf{r}) \psi_{j}^{\dagger}\left(\mathbf{r}^{\prime}\right) \frac{1}{\left|\mathbf{r}-\mathbf{r}^{\prime}\right|} \psi_{k}(\mathbf{r}) \psi_{l}\left(\mathbf{r}^{\prime}\right)
$$

and the quantity $\epsilon_{i}$ in Eq. (9) is the eigenvalue of the Dirac equation. The third term in (10) is a c-number and provides an additive constant to the energy of the atom.

The all-order SD wave function given by Eq. (7) includes only terms that are linear in the excitation coefficients. In the present work, we take into account all non-linear terms that arise from the single and double excitations. Out of all possible non-linear terms, only six terms, $\frac{1}{2} S_{1}^{2}, S_{1} S_{2}, \frac{1}{6} S_{1}^{3}, \frac{1}{2} S_{2}^{2}, \frac{1}{2} S_{1}^{2} S_{2}$, and $\frac{1}{24} S_{1}^{4}$ contribute to the single-double equations. Explicitly, the non-linear terms contributing to the core single-double equations are $\frac{1}{2} S_{1 c}^{2}, S_{1 c} S_{2 c}, \frac{1}{6} S_{1 c}^{3}, \frac{1}{2} S_{2 c}^{2}, \frac{1}{2} S_{1 c}^{2} S_{2 c}$, and $\frac{1}{24} S_{1 c}^{4}$ and the non-linear terms contributing to the valence singledouble equations are $S_{1 c} S_{1 v},\left\{S_{1 v} S_{2 c}, S_{1 c} S_{2 v}\right\}, \frac{1}{2} S_{1 c}^{2} S_{1 v}$, $S_{2 c} S_{2 v},\left\{S_{1 v} S_{1 c} S_{2 c}, \frac{1}{2} S_{1 c}^{2} S_{2 v}\right\}$, and $\frac{1}{6} S_{1 c}^{3} S_{1 v}$.

First, we consider the contributions from the nonlinear core terms. The first three non-linear core terms

$$
\begin{aligned}
& T_{1}=\frac{1}{2} S_{1 c}^{2}=\frac{1}{2} \sum_{r s c d} \rho_{r c} \rho_{s d} a_{r}^{\dagger} a_{s}^{\dagger} a_{d} a_{c} \\
& T_{2}=S_{1 c} S_{2 c}=\frac{1}{2} \sum_{r s t c d e} \rho_{t e} \rho_{r s c d} a_{r}^{\dagger} a_{s}^{\dagger} a_{t}^{\dagger} a_{e} a_{d} a_{c}, \\
& T_{3}=\frac{1}{6} S_{1 c}^{3}=\frac{1}{6} \sum_{r s t c d e} \rho_{t e} \rho_{r c} \rho_{s d} a_{r}^{\dagger} a_{s}^{\dagger} a_{t}^{\dagger} a_{e} a_{d} a_{c}
\end{aligned}
$$

contribute to equations for both single and double excitation coefficients, while the last three terms

$$
\begin{aligned}
T_{4} & =\frac{1}{2} S_{2 c}^{2}=\frac{1}{8} \sum_{\text {rstucdef }} \rho_{\text {rscd }} \rho_{\text {tuef }} a_{r}^{\dagger} a_{s}^{\dagger} a_{t}^{\dagger} a_{u}^{\dagger} a_{f} a_{e} a_{d} a_{c},(14) \\
T_{5} & =\frac{1}{2} S_{1 c}^{2} S_{2 c} \\
& =\frac{1}{4} \sum_{r s t u c d e f} \rho_{t e} \rho_{u f} \rho_{r s c d} a_{r}^{\dagger} a_{s}^{\dagger} a_{t}^{\dagger} a_{u}^{\dagger} a_{f} a_{e} a_{d} a_{c}, \\
T_{6} & =\frac{1}{24} S_{1 c}^{4}=\frac{1}{24} \sum_{r s t u c d e f} \rho_{r c} \rho_{s d} \rho_{t e} \rho_{u f} a_{r}^{\dagger} a_{s}^{\dagger} a_{t}^{\dagger} a_{u}^{\dagger} a_{f} a_{e} a_{d} a_{c}
\end{aligned}
$$

contribute to the equation for the double excitation coefficients only.

The right-hand side of the single-double equations is obtained by operating on the non-linear terms above with the two-particle interaction operator

$$
G=\frac{1}{2} \sum_{i j k l} g_{i j k l}\left[a_{i}^{\dagger} a_{j}^{\dagger} a_{l} a_{k}\right]
$$

according to Eq. (10).

To derive the equation for the core single-excitation coefficients, we extract those terms in $G T_{k}(k=1,2,3)$ that are proportional to $a_{m}^{\dagger} a_{a} a_{v}^{\dagger}$. To derive the equation for the core double-excitation coefficients, we extract the terms in $G T_{k}(k=1 \cdots 6)$ that are proportional to $\frac{1}{2} a_{m}^{\dagger} a_{n}^{\dagger} a_{b} a_{a} a_{v}^{\dagger}$. In all cases, we drop terms corresponding to disconnected diagrams. Below, we use the notations $G T_{k}^{s}$ and $G T_{k}^{d}$ to designate contribution of the corresponding terms to the single or double excitation equations, respectively. For clarity, we give the contributions from all terms separately.

The equation for the core single-excitation coefficients becomes

$$
\left(\epsilon_{a}-\epsilon_{m}\right) \rho_{m a}=\mathrm{SD}+G T_{1}^{s}+G T_{2}^{s}+G T_{3}^{s},
$$


where $\epsilon_{i}$ is the one-body DF energy for the state $i, \mathrm{SD}$ is the contribution from the linear coupled-cluster terms given in 24], and the contributions of the non-linear terms are

$$
\begin{aligned}
G T_{1}^{s} & =\sum_{d r s} \tilde{g}_{m d r s} \rho_{r a} \rho_{s d}-\sum_{c d s} \tilde{g}_{c d a s} \rho_{m c} \rho_{s d} \\
G T_{2}^{s} & =-\sum_{c d r s} \tilde{g}_{c d s r} \rho_{r s d a} \rho_{m c}-\sum_{c d r s} \tilde{g}_{c d s r} \rho_{s m c d} \rho_{r a} \\
& +\sum_{c d r s} \tilde{g}_{c d r s} \tilde{\rho}_{r m c a} \rho_{s d}, \\
G T_{3}^{s} & =-\sum_{c d r s} \tilde{g}_{c d s r} \rho_{m c} \rho_{r d} \rho_{s a} .
\end{aligned}
$$

We used the notation $\tilde{g}_{m n a b}=g_{m n a b}-g_{m n b a}$ and $\tilde{\rho}_{m n a b}=\rho_{m n a b}-\rho_{m n b a}$ in the above formulas. is

The equation for the core double-excitation coefficients

$$
\begin{aligned}
& \left(\epsilon_{a}+\epsilon_{b}-\epsilon_{m}-\epsilon_{n}\right) \rho_{m n a b}=\mathrm{SD} \\
& \quad+G T_{1}^{d}+G T_{2}^{d}+G T_{3}^{d}+G T_{4}^{d}+G T_{5}^{d}+G T_{6}^{d}
\end{aligned}
$$

where

$$
\begin{aligned}
& G T_{1}^{d}=\sum_{r s} g_{m n r s} \rho_{r a} \rho_{s b}+\sum_{c d} g_{c d a b} \rho_{m c} \rho_{n d} \\
& -\left[\sum_{d r} \tilde{g}_{m d a r} \rho_{r b} \rho_{n d}+\left(\begin{array}{c}
a \leftrightarrow b \\
m \leftrightarrow n
\end{array}\right)\right], \\
& G T_{2}^{d}=\left[-\sum_{c d r} \tilde{g}_{c d r b} \rho_{n d} \tilde{\rho}_{r m c a}-\sum_{c d r} \tilde{g}_{c d a r} \rho_{r d} \rho_{m n c b}\right. \\
& +\sum_{c d r} g_{c d r a} \rho_{r b} \rho_{n m c d}+\sum_{c r s} \tilde{g}_{n c r s} \rho_{r b} \tilde{\rho}_{s m c a} \\
& +\sum_{c r s} \tilde{g}_{n c r s} \rho_{s c} \rho_{m r a b}-\sum_{c r s} g_{n c r s} \rho_{m c} \rho_{s r a b} \\
& \left.+\left(\begin{array}{c}
a \leftrightarrow b \\
m \leftrightarrow n
\end{array}\right)\right] \\
& G T_{3}^{d}=\left[\sum_{c d r} g_{c d a r} \rho_{n d} \rho_{m c} \rho_{r b}-\sum_{c r s} g_{m c r s} \rho_{n c} \rho_{r a} \rho_{s b}\right. \\
& \left.+\left(\begin{array}{c}
a \leftrightarrow b \\
m \leftrightarrow n
\end{array}\right)\right] \\
& G T_{4}^{d}=\sum_{c d t u} g_{c d t u} \rho_{t u a b} \rho_{m n c d}+\sum_{c d t u} \tilde{g}_{c d t u} \tilde{\rho}_{m t a c} \tilde{\rho}_{u n d b} \\
& -\left[\sum_{c d t u} \tilde{g}_{c d t u}\left(\rho_{t u b d} \rho_{m n a c}+\rho_{m u c d} \rho_{n t b a}\right)\right. \\
& \left.+\left(\begin{array}{c}
a \leftrightarrow b \\
m \leftrightarrow n
\end{array}\right)\right],
\end{aligned}
$$

$$
\begin{gathered}
G T_{5}^{d}=\sum_{c d t u} g_{c d t u}\left(\rho_{t a} \rho_{u b} \rho_{m n c d}+\rho_{m c} \rho_{n d} \rho_{t u a b}\right) \\
-\left[\sum_{c d t u} \tilde{g}_{c d u t} \rho_{t b} \rho_{u c} \rho_{m n a d}+\sum_{c d t u} \tilde{g}_{c d t u} \rho_{t c} \rho_{n d} \rho_{m u a b}\right. \\
\left.\left.+\sum_{c d t u} \tilde{g}_{c d t u} \rho_{t b} \rho_{n c} \tilde{\rho}_{m u a d}\right)+\left(\begin{array}{c}
a \leftrightarrow b \\
m \leftrightarrow n
\end{array}\right)\right] \\
G T_{6}^{d}=\sum_{c d t u} g_{c d t u} \rho_{t a} \rho_{u b} \rho_{m c} \rho_{n d} .
\end{gathered}
$$

All non-linear contributions to double-excitation coefficients are symmetrized to preserve the property $\rho_{\text {mnab }}=$ $\rho_{n m b a}$.

Only one non-linear term, $G T_{1}=\frac{1}{2} G S_{1 c}^{2}$, contributes to the equation for the core correlation energy:

$$
\delta E_{c}=\delta E_{c}^{\mathrm{SD}}+\sum_{a b m n} \frac{1}{2} \tilde{g}_{a b m n} \rho_{m a} \rho_{n b},
$$

where $\delta E_{c}^{\mathrm{SD}}$ is the core correlation energy obtained with linearized SD wave function (7):

$$
\delta E_{c}^{\mathrm{SD}}=\frac{1}{2} \sum_{m n a b} g_{a b m n} \tilde{\rho}_{m n a b} .
$$

We note that the summation over each index, for example $i$, involves summing over the principal quantum number $n_{i}$, the relativistic angular momentum quantum number $\kappa_{i}$, and the magnetic quantum number $m_{i}$. The sum over the magnetic quantum numbers is carried out analytically and the final formulas are given in Appendix A

The equations for the valence excitation coefficients $\rho_{m v}$ and $\rho_{m n v b}$ are identical to the core equations given by Eqs. (18,28) with replacement of index $a$ by index $v$ and addition of the valence correlation energy $\delta E_{v}$ into the parenthesis on the left-hand side of both equations, i.e.

$$
\begin{gathered}
\left(\epsilon_{v}-\epsilon_{m}+\delta E_{v}\right) \rho_{m v}=\mathrm{SD}+\left(G T_{1}^{s}+G T_{2}^{s}+G T_{3}^{s}\right)_{a \rightarrow v} \\
\left(\epsilon_{v}+\epsilon_{b}-\epsilon_{m}-\epsilon_{n}+\delta E_{v}\right) \rho_{m n v b}=\mathrm{SD}+\left(G T_{1}^{d}\right. \\
\left.+G T_{2}^{d}+G T_{3}^{d}+G T_{4}^{d}+G T_{5}^{d}+G T_{6}^{d}\right)_{a \rightarrow v} \cdot
\end{gathered}
$$

The valence correlation energy $\delta E_{v}$ is given by

$$
\begin{aligned}
\delta E_{v} & =\delta E_{v}^{\mathrm{SD}}-\sum_{c d t} \tilde{g}_{c d v t} \rho_{t d} \rho_{v c}+\sum_{d t u} \tilde{g}_{v d t u} \rho_{t v} \rho_{u d} \\
& -\sum_{c d t u} \tilde{g}_{c d u t} \rho_{v c} \rho_{u t v d}-\sum_{c d t u} \tilde{g}_{c d u t} \rho_{t v} \rho_{u v c d} \\
& +\sum_{c d t u} \tilde{g}_{c d t u} \tilde{\rho}_{v t v c} \rho_{u d}-\sum_{c d t u} \tilde{g}_{c d u t} \rho_{t d} \rho_{u v} \rho_{v c} .
\end{aligned}
$$

The term $\delta E_{v}^{\mathrm{SD}}$ represents the expression for the valence correlation energy without the non-linear terms [24]:

$\delta E_{v}^{\mathrm{SD}}=\sum_{m a} \tilde{g}_{v a v m} \rho_{m a}+\sum_{m a b} g_{a b v m} \tilde{\rho}_{m v a b}+\sum_{m n b} g_{v b m n} \tilde{\rho}_{m n v b}$. 
The non-linear contributions to the valence correlation energy arise from the $S_{1 c} S_{1 v},\left\{S_{1 v} S_{2 c}, S_{1 c} S_{2 v}\right\}$, and $\frac{1}{2} S_{1 c}^{2} S_{1 v}$ terms.

We solve the SD equations using a finite basis set. Each orbital wave function is represented as a linear combination of the B-splines. We consider a radial grid of 250 points within a sphere of radius 100 a.u. We include 35 out of 40 basis orbitals for each angular momentum and include all partial waves with $l \leq 6$ in our calculations. A detailed description of the B-spline method is given in Ref. [30]. We treat the non-linear terms on the same footing with the linear terms, i.e. all the linear and nonlinear terms are iterated together. First, the equations for the single core and double core excitation coefficients are iterated until the core correlation energy given by Eq. (29) converges to relative accuracy $\epsilon=10^{-5}$. Then, the valence equations are iterated until the valence correlation energy given by Eq. (32) converges to the relative accuracy $\epsilon$. Atomic properties can be evaluated once the values of the excitation coefficients are known, as briefly described below.

Matrix elements of a one-body operator $Z=$ $\sum_{i j} Z_{i j} a_{i}^{\dagger} a_{j}$ are determined using the formula

$$
Z_{w v}=\frac{\left\langle\Psi_{w}|Z| \Psi_{v}\right\rangle}{\sqrt{\left\langle\Phi_{v} \mid \Phi_{v}\right\rangle\left\langle\Phi_{w} \mid \Phi_{w}\right\rangle}}
$$

Substituting the expression for the wave function from Eq. (7) in the above equation and simplifying, one finds

$$
Z_{w v}=\delta_{w v} Z_{\mathrm{core}}+\frac{Z_{\mathrm{val}}}{\sqrt{\left(1+N_{v}\right)\left(1+N_{w}\right)}},
$$

where $Z_{\text {core }}, Z_{\text {val }}, N_{v}$, and $N_{w}$ are linear or quadratic functions of the single and double excitation coefficients written out in Refs. [1, 24].

In general, the non-linear terms coming from expanding the exponent in the $\mathrm{CC}$ wave function also contribute to the expressions to matrix elements. Even at the CCSD truncation level, one encounters an infinite number of such contributions. A rigorous method of partial summation (dressing) of the resulting series was devised in Ref. [31]. The method is built upon expanding a product of cluster amplitudes into a sum of $n$-body insertions. Although in the present paper we do not include these direct non-linear contributions to matrix elements, calculations [20] show that dressing may contribute as much as a few $0.1 \%$ to hyperfine constants in Cs.

\section{RESULTS AND DISCUSSION}

\section{A. Energies}

Table I shows a detailed breakdown of contributions from non-linear terms to the removal energies of the alkali-metal atoms $\mathrm{Li}, \mathrm{Na}, \mathrm{K}, \mathrm{Rb}$, and $\mathrm{Cs}$. To illustrate the relative importance of the various terms, we
TABLE I: Contributions of the non-linear (NL) terms to removal energies for $\mathrm{Li}, \mathrm{Na}, \mathrm{K}, \mathrm{Rb}$, and Cs. A detailed description of all contributions is given in text. SD designates the correlation corrections to the energies calculated using the SD

\begin{tabular}{|c|c|c|c|}
\hline $\mathrm{Li}$ & $2 s_{1 / 2}$ & $2 p_{1 / 2}$ & $2 p_{3 / 2}$ \\
\hline $\mathrm{SD}$ & 406.0 & 352.1 & 352.0 \\
\hline Core NL terms & 0.9 & 0.1 & 0.1 \\
\hline$S_{2 c} S_{2 v}$ & -5.1 & -4.2 & -4.2 \\
\hline$S_{1 c} S_{1 v},\left\{S_{1 v} S_{2 c}, S_{1 c} S_{2 v}\right\}$ & -2.6 & -0.9 & -0.9 \\
\hline Other valence NL SD terms & 0.0 & 0.0 & 0.0 \\
\hline Total & 399.2 & 347.1 & 347.0 \\
\hline $\mathrm{Na}$ & $3 s_{1 / 2}$ & $3 p_{1 / 2}$ & $3 p_{3 / 2}$ \\
\hline $\mathrm{SD}$ & 1483.3 & 462.0 & 459.8 \\
\hline Core NL terms & 0.5 & 2.6 & 2.5 \\
\hline$S_{2 c} S_{2 v}$ & -44.3 & -15.3 & -15.3 \\
\hline$S_{1 c} S_{1 v},\left\{S_{1 v} S_{2 c}, S_{1 c} S_{2 v}\right\}$ & -23.8 & -10.2 & -10.1 \\
\hline Other valence NL SD terms & 0.0 & 0.0 & 0.0 \\
\hline Total & 1415.6 & 439.1 & 436.9 \\
\hline $\mathrm{K}$ & $4 s_{1 / 2}$ & $4 p_{1 / 2}$ & $4 p_{3 / 2}$ \\
\hline $\mathrm{SD}$ & 2869.7 & 1114.3 & 1100.5 \\
\hline Core NL terms & 26.8 & 11.3 & 11.2 \\
\hline$S_{2 c} S_{2 v}$ & -142.5 & -59.1 & -58.5 \\
\hline$S_{1 c} S_{1 v},\left\{S_{1 v} S_{2 c}, S_{1 c} S_{2 v}\right\}$ & -62.9 & -34.4 & -34.3 \\
\hline Other valence NL SD terms & 0.1 & 0.1 & 0.1 \\
\hline Total & 2691.2 & 1032.2 & 1019.1 \\
\hline $\mathrm{Rb}$ & $5 s_{1 / 2}$ & $5 p_{1 / 2}$ & $5 p_{3 / 2}$ \\
\hline $\mathrm{SD}$ & 3423.2 & 1301.1 & 1236.3 \\
\hline Core NL terms & 31.7 & 13.5 & 13.1 \\
\hline$S_{2 c} S_{2 v}$ & -185.4 & -76.7 & -73.2 \\
\hline$S_{1 c} S_{1 v},\left\{S_{1 v} S_{2 c}, S_{1 c} S_{2 v}\right\}$ & -105.0 & -47.9 & -46.3 \\
\hline Other valence NL SD terms & 0.3 & 0.1 & 0.1 \\
\hline Total & 3164.8 & 1190.1 & 1130.0 \\
\hline Cs & $6 s_{1 / 2}$ & $6 p_{1 / 2}$ & $6 p_{3 / 2}$ \\
\hline $\mathrm{SD}$ & 3881.5 & 1618.7 & 1442.3 \\
\hline Core NL terms & 44.3 & 18.1 & 16.8 \\
\hline$S_{2 c} S_{2 v}$ & -224.4 & -107.3 & -96.3 \\
\hline$S_{1 c} S_{1 v},\left\{S_{1 v} S_{2 c}, S_{1 c} S_{2 v}\right\}$ & -162.4 & -74.6 & -68.3 \\
\hline Other valence NL SD terms & 0.9 & 0.4 & 0.4 \\
\hline Total & 3539.9 & 1455.3 & 1294.9 \\
\hline
\end{tabular}
method. All results are in $\mathrm{cm}^{-1}$.

conducted five separate calculations for each atom. Each subsequent calculation includes all terms in the previous calculation together with additional terms, the effect of which is being determined. For clarity, we describe each of the calculations below.

1. Linearized SD calculation, with all non-linear terms omitted. The results of this calculation are listed in the rows labeled "SD".

2. All non-linear terms are included in the core equations only, all non-linear valence terms are omitted. The differences of those values and the SD results are listed in rows labeled "Core NL terms".

3. All non-linear terms are included in the core equa- 
tions and the quadratic term $S_{2 c} S_{2 v}$ is included in the valence equations. The differences with the calculation (2) give the contributions from the nonlinear valence term $S_{2 c} S_{2 v}$ and are listed in the rows labeled accordingly.

4. All non-linear terms are included in the core equations, and all remaining quadratic terms are included in the valence equations. The differences between those values and the results of the calculation (3) give the contributions of the $S_{1 c} S_{1 v}$ and $\left\{S_{1 v} S_{2 c}, S_{1 c} S_{2 v}\right\}$ quadratic valence terms.

5. Final calculation: all non-linear terms are included in the core and valence equations. The results of this calculation are listed in the rows labeled "Total". The differences between those values and the results of the calculation (4) give the contributions of the cubic and quartic non-linear terms that are listed in rows labeled "Other valence NL SD terms".

In all of the cases considered here, the addition of the non-linear terms results in a decrease in the correlation contributions to the removal energies. From Table II. we see that while the contribution of the core nonlinear terms to the removal energies is negligible compared to contribution of the valence non-linear terms for the ground states of $\mathrm{Na}$, it becomes significant (over 10\% of the total NL contribution and about $1 \%$ of the total correlation energy) for all states of $\mathrm{K}, \mathrm{Rb}$, and Cs considered here. Furthermore, the contribution from core NL terms is opposite in sign to that from the valence non-linear contribution.

The $S_{2 c} S_{2 v}$ term gives the dominant nonlinear contribution, as expected. More than half of the contribution from non-linear terms to the removal energy arises from this term. However, contributions from other quadratic terms, $S_{1 c} S_{1 v}$ and $\left\{S_{1 v} S_{2 c}, S_{1 c} S_{2 v}\right\}$, are also significant. Finally, the contributions from terms $\frac{1}{2} S_{1 c}^{2} S_{1 v}$, $\left\{S_{1 v} S_{1 c} S_{2 c}, \frac{1}{2} S_{1 c}^{2} S_{2 v}\right\}$, and $\frac{1}{6} S_{1 c}^{3} S_{1 v}$, i.e. terms that are cubic or quartic in the excitation coefficients are negligible. For $\mathrm{Li}$ and $\mathrm{Na}$, contributions from these terms are essentially zero; for $\mathrm{K}, \mathrm{Rb}$, and $\mathrm{Cs}$, the contributions are less than $0.5 \%$ of the total from all non-linear terms. The breakdown of contributions from the valence NL terms is essentially identical for all states of $\mathrm{Na}, \mathrm{K}$, $\mathrm{Rb}$, and Cs under consideration. The total contribution of the NL terms to the correlation energies of the lowest three states of $\mathrm{Na}, \mathrm{K}, \mathrm{Rb}$, and $\mathrm{Cs}$ is remarkably large; it is about $1.5 \%$ of the linear SD correlation energy for $\mathrm{Li}$, $5 \%$ for $\mathrm{Na}$, and $9 \%$ for Cs.

In Table II we compare of our results for the correlation with the linearized all-order $\mathrm{SD}(\mathrm{pT})$ calculations of Ref. [2], CCSD calculations of Ref. [14], the CCSD(T) calculations of Ref. [18] and with the experimental energies [32]. The values in the rows labeled "SD" are the sum of lowest-order (DF) energies, the SD contributions given in Table I and the extrapolated contributions of
TABLE II: Comparison of the all-order removal energies with the theoretical results obtained by the coupled-cluster method [14, 18] and experiment. All the values are in $\mathrm{cm}^{-1}$.

\begin{tabular}{|c|c|c|c|}
\hline $\mathrm{Li}$ & $2 s_{1 / 2}$ & $2 p_{1 / 2}$ & $2 p_{3 / 2}$ \\
\hline SD & 43494 & 28586 & 28585 \\
\hline CCSD & 43488 & 28581 & 28580 \\
\hline Expt. [32] & 43487 & 28584 & 28583 \\
\hline $\mathrm{Na}$ & $3 s_{1 / 2}$ & $3 p_{1 / 2}$ & $3 p_{3 / 2}$ \\
\hline $\mathrm{SD}$ & 41444 & 24495 & 24476 \\
\hline CCSD & 41376 & 24472 & 24453 \\
\hline $\mathrm{SD}[2]$ & 41447 & 24494 & 24477 \\
\hline CCSD[14] & 41352 & 24465 & 24447 \\
\hline Expt. [32] & 41449 & 24493 & 24476 \\
\hline $\mathrm{K}$ & $4 s_{1 / 2}$ & $4 p_{1 / 2}$ & $4 p_{3 / 2}$ \\
\hline $\mathrm{SD}$ & 35258 & 22219 & 22066 \\
\hline CCSD & 35080 & 22044 & 21984 \\
\hline $\mathrm{SD}[2]$ & 34962 & 22023 & 21966 \\
\hline CCSD[14] & 35028 & 22016 & 21957 \\
\hline Expt. [32] & 35010 & 22025 & 21967 \\
\hline $\mathrm{Rb}$ & $5 s_{1 / 2}$ & $5 p_{1 / 2}$ & $5 p_{3 / 2}$ \\
\hline $\mathrm{SD}$ & 34021 & 21241 & 20994 \\
\hline CCSD & 33762 & 21130 & 20888 \\
\hline $\mathrm{SD}[2]$ & 33649 & 21111 & 20875 \\
\hline CCSD[14] & 33721 & 21117 & 20877 \\
\hline CCSD [18] & 33603 & 21080 & 20831 \\
\hline Expt. [32] & 33691 & 21112 & 20874 \\
\hline Cs & $6 s_{1 / 2}$ & $6 p_{1 / 2}$ & $6 p_{3 / 2}$ \\
\hline $\mathrm{SD}$ & 31871 & 20421 & 19842 \\
\hline CCSD & 31529 & 20258 & 19695 \\
\hline $\mathrm{SD}[2]$ & 31262 & 20204 & 19652 \\
\hline CCSD[14] & 31443 & 20217 & 19669 \\
\hline CCSD[18] & 31250 & 20137 & 19574 \\
\hline Expt. [32] & 31407 & 20228 & 19674 \\
\hline
\end{tabular}

the higher partial waves $\left(\mathrm{E}_{\text {extrap }}\right)$; the values in the rows labeled "CCSD" are the sums of the DF, SD, non-linear contributions, and $\mathrm{E}_{\text {extrap }}$. The CCSD values should agree with the calculation of Ref. [14] within the numerical uncertainties of the calculations. Significant differences between all-order SD results and CCSD results were noted earlier in Ref. [2], indicating that NL terms may be large. In this work, we find a good agreement between our CCSD values and the results of Ref. [14]. The remaining discrepancies can be explained by the differences in some numerical details of the calculations. In our calculations, we truncated the number of partial waves included in all sums over excited states at $l_{\max }=6$. Contributions from partial waves with $l>6$ are extrapolated in second order (see [3] for details of the extrapolation procedure). The resulting correction increase for heavier alkali-metal atoms and is about $0.1 \%$ of the total energy of $6 s$ state in Cs. The differences between our values and those of Ref. 14] are of the same order of magnitude as the $\mathrm{E}_{\text {extrap }}$ contributions. 
The SD approximation omits contributions to energies of third order in perturbation theory that arise from triples excitations. These missing third-order terms were included in the calculation of Ref. 2]. The size of this terms is approximately given by the differences between values listed in rows "SD" and "SD[2]". The calculation of Ref. [14] omits these terms entirely. Interestingly, these terms are of nearly the same magnitude as the NL terms and of the same sign. As a result, both calculation of Ref. [2], that omitted NL terms, and of Ref. [14], that omitted missing third-order terms, were in quite good agreement with experiment. It was shown in [19, 20], that actual iterated triple contribution significantly differs from the third-order values. For this reason, and to make a clear comparison with previous CCSD results, we omit all triples contributions in this calculation. For Rb and $\mathrm{Cs}$, we also list values from the $\operatorname{CCSD}(\mathrm{T})$ calculations of Ref. [18] in the rows labeled "CCSD 18]" that include contributions from triple excitations, but omit the odd-parity channels.

\section{B. Reduced electric-dipole matrix elements}

Table III gives the detailed breakdown of the contributions of the non-linear terms to the reduced electricdipole matrix elements for $\mathrm{Li}, \mathrm{Na}, \mathrm{K}, \mathrm{Rb}$, and $\mathrm{Cs}$. This table is structured in exactly the same way as Table [1 the only exception is that we added lowest-order DF value to the linearized SD values in rows labeled "SD". The breakdown of the non-linear terms is identical to the breakdown for correlation energies. The major contribution comes from the term $S_{2 c} S_{2 v}$ as in the case of the removal energies. Also, there is almost no contribution from terms which are cubic or quartic in the excitation coefficients. The core non-linear terms and the cubic and quartic valence non-linear terms decrease the E1 reduced matrix elements, while the quadratic valence non-linear terms increase these values. The only exception to the statement above is the contribution of the core non-linear terms for $\mathrm{Na}$ which behaves in the opposite manner. Since the contribution of the quadratic valence non-linear terms outweigh the contribution of all the other terms, the ultimate effect is an increase in values of the reduced electric-dipole matrix elements. As in the case of the correlation energies, the contribution of the NL terms is rather large, especially for heavy alkalies where it reaches $1.5 \%$. The comparison of our results with the experiment [33, 34] is also given in Table III] Addition of non-linear terms results in a deterioration of the agreement with experimental values as expected from [19, 20] owing to significant cancellation between the valence NL and valence triple terms. A very interesting finding of this work is relatively large contribution of the core non-linear terms for heavy alkalies. While the core non-linear term is entirely negligible for $\mathrm{Na}$, it is found to be $0.2 \%$ for Cs.
TABLE III: Contributions of the non-linear terms to the $n s_{1 / 2}-n p_{1 / 2}$ and $n s_{1 / 2}-n p_{3 / 2}$ reduced electric-dipole matrix elements for $\mathrm{Li}, \mathrm{Na}, \mathrm{K}, \mathrm{Rb}$, and $\mathrm{Cs}$. The final values are compared with experimental results. All values are given in atomic units (ea $a_{0}$, where $a_{0}$ is the Bohr radius).

\begin{tabular}{|c|c|c|}
\hline $\mathrm{Li}$ & $2 s_{1 / 2}-2 p_{1 / 2}$ & $2 s_{1 / 2}-2 p_{3 / 2}$ \\
\hline $\mathrm{SD}$ & 3.31654 & 4.69033 \\
\hline Core NL terms & -0.00007 & -0.00008 \\
\hline$S_{2 c} S_{2 v}$ & 0.00063 & 0.00088 \\
\hline$S_{1 c} S_{1 v},\left\{S_{1 v} S_{2 c}, S_{1 c} S_{2 v}\right\}$ & 0.00020 & 0.00028 \\
\hline Other valence NL SD terms & 0.00000 & 0.00000 \\
\hline Total & 3.31730 & 4.69141 \\
\hline Expt. [33] & $3.317(4)$ & $4.689(5)$ \\
\hline $\mathrm{Na}$ & $3 s_{1 / 2}-3 p_{1 / 2}$ & $3 s_{1 / 2}-3 p_{3 / 2}$ \\
\hline SD & 3.53099 & 4.99314 \\
\hline Core NL terms & 0.00005 & 0.00006 \\
\hline$S_{2 c} S_{2 v}$ & 0.00487 & 0.00690 \\
\hline$S_{1 c} S_{1 v},\left\{S_{1 v} S_{2 c}, S_{1 c} S_{2 v}\right\}$ & 0.00211 & 0.00297 \\
\hline Other valence NL SD terms & 0.00000 & 0.00000 \\
\hline Total & 3.53802 & 5.00307 \\
\hline Expt. [33] & $3.5246(23)$ & $4.9838(34)$ \\
\hline $\mathrm{K}$ & $4 s_{1 / 2}-4 p_{1 / 2}$ & $4 s_{1 / 2}-4 p_{3 / 2}$ \\
\hline SD & 4.09820 & 5.79392 \\
\hline Core NL terms & -0.00474 & -0.00669 \\
\hline$S_{2 c} S_{2 v}$ & 0.02261 & 0.03198 \\
\hline$S_{1 c} S_{1 v},\left\{S_{1 v} S_{2 c}, S_{1 c} S_{2 v}\right\}$ & 0.00873 & 0.01231 \\
\hline Other valence NL SD terms & -0.00002 & -0.00003 \\
\hline Total & 4.12478 & 5.83149 \\
\hline Expt. [33] & $4.102(5)$ & $5.800(8)$ \\
\hline $\mathrm{Rb}$ & $5 s_{1 / 2}-5 p_{1 / 2}$ & $5 s_{1 / 2}-5 p_{3 / 2}$ \\
\hline $\mathrm{SD}$ & 4.22005 & 5.95527 \\
\hline Core NL terms & -0.00649 & -0.00913 \\
\hline$S_{2 c} S_{2 v}$ & 0.03230 & 0.04564 \\
\hline$S_{1 c} S_{1 v},\left\{S_{1 v} S_{2 c}, S_{1 c} S_{2 v}\right\}$ & 0.01533 & 0.02156 \\
\hline Other valence NL SD terms & -0.00004 & -0.00006 \\
\hline Total & 4.26115 & 6.01328 \\
\hline Expt. [33] & $4.231(3)$ & $5.977(4)$ \\
\hline Cs & $6 s_{1 / 2}-6 p_{1 / 2}$ & $6 s_{1 / 2}-6 p_{3 / 2}$ \\
\hline $\mathrm{SD}$ & 4.48157 & 6.30391 \\
\hline Core NL terms & -0.01057 & -0.01482 \\
\hline$S_{2 c} S_{2 v}$ & 0.04585 & 0.06437 \\
\hline$S_{1 c} S_{1 v},\left\{S_{1 v} S_{2 c}, S_{1 c} S_{2 v}\right\}$ & 0.02762 & 0.03865 \\
\hline Other valence NL SD terms & -0.00015 & -0.00021 \\
\hline Total & 4.54432 & 6.39190 \\
\hline Expt. [34] & $4.4890(65)$ & $6.3238(73)$ \\
\hline
\end{tabular}

\section{Hyperfine constants}

We calculate the magnetic-dipole hyperfine constants $A$ for the $n s_{1 / 2}$ ground states and the $n p_{1 / 2}, n p_{3 / 2}$ excited states of $\mathrm{Li}, \mathrm{Na}, \mathrm{K}, \mathrm{Rb}$, and $\mathrm{Cs}$. The nuclear magnetization density is described by a Fermi distribution with half-density radius $c$ and 90\%-10\% falloff thickness $t=2.3 \mathrm{fm}$. Table IV lists values of the parameter $c(\mathrm{fm})$ used for magnetization distribution and the gyromagnetic ratio $g_{I}$ for each of the alkali-metal atoms. The 
TABLE IV: Values of the Fermi half-density $c(\mathrm{fm})$ parameter used for magnetization distribution and gyromagnetic ratio $g_{I}$ (in units of the nuclear magneton) which were used to calculate hyperfine constants.

\begin{tabular}{lcl}
\hline \hline Atom & $c$ & \multicolumn{1}{c}{$g_{I}$} \\
\hline${ }^{7} \mathrm{Li}$ & 1.7995 & 2.17093 \\
${ }^{23} \mathrm{Na}$ & 2.8853 & 1.4784 \\
${ }^{39} \mathrm{~K}$ & 3.6108 & 0.260993 \\
${ }^{85} \mathrm{Rb}$ & 4.8708 & 0.54136 \\
${ }^{133} \mathrm{Cs}$ & 5.6748 & 0.737886 \\
\hline \hline
\end{tabular}

nuclear magnetic moments were taken from Ref. [35] and weighted averages were considered when more than one value was present.

Contributions of the various non-linear terms to the hyperfine constants are given in Table $\mathrm{V}$. The comparison of our results with experiment [36, 37, 38, 39, 40, 41, 42, 43] is also given. The structure of Table $\mathrm{V}$ is identical to that of Table III The "SD" values are the sum of the lowest-order DF values and correlation correction calculated in the absence of the NL terms. Addition of the non-linear terms resulted in a decrease in the values of the hyperfine constants in comparison to the linearized $\mathrm{SD}$ values. The NL core terms and the cubic and quartic valence non-linear terms contribute with a positive sign to the hyperfine constants, while the quadratic valence non-linear terms contribute with negative sign for $\mathrm{K}, \mathrm{Rb}$, and $\mathrm{Cs}$. For $\mathrm{Na}$ and $\mathrm{Li}$, all core and valence nonlinear terms contribute with the same sign. The most significant difference between the NL contributions to the hyperfine constants and to the removal energies and E1 matrix elements is that the contribution of the $S_{2 c} S_{2 v}$ terms is smaller than the contribution of the other three quadratic valence non-linear terms $S_{1 c} S_{1 v},\left\{S_{1 v} S_{2 c}\right.$, and $\left.S_{1 c} S_{2 v}\right\}$ for the ground states of $\mathrm{Na}, \mathrm{K}, \mathrm{Rb}$, and Cs. The breakdown is also different for the $n s, n p_{1 / 2}$, and $n p_{3 / 2}$ hyperfine constants while it is very uniform for all energies and E1 matrix elements considered here. The contributions of the core NL terms are particularly large for the hyperfine constants, almost $1 \%(!)$ of the total values for Cs.

\section{CONCLUSION}

We have extended the relativistic SD method to include all non-linear terms at the SD level. The effect of the non-linear terms on the removal energies, hyperfine constants, and electric-dipole matrix elements of the alkali-metal atoms from Li to Cs was systematically investigated. In particular, five different calculations were carried out to establish the importance of the various contributions for each alkali-metal atom. The effect of the core non-linear terms was found to be not negligible for heavier alkalies, reaching nearly $1 \%$ of the total values of the Cs hyperfine constants. Among other terms, the
$S_{2}^{2}$ term was found to be dominant for removal energies and electric-dipole matrix elements with other quadratic terms being also significant. In the case of the hyperfine constants, the contributions of the other quadratic terms exceeded that of the $S_{2}^{2}$ term for most cases. The contribution of the cubic and quartic terms was found to be negligible in all cases considered in the present work. Inclusion of non-linear terms in the single-double all-order method is a significant step toward further development of high-precision methodologies for the calculation of the atomic properties.

\section{ACKNOWLEDGMENTS}

The work of R.P. and M.S.S. was supported in part by National Science Foundation Grant No. PHY-0457078. The work of W.R.J. was supported in part by National Science Foundation Grant No. PHY-0456828. The work of A.D. was supported in part by a National Science Foundation Grant No. PHY-0354876 and by a NIST Precision Measurement grant. The work of S.G.P. was supported in part by the Russian Foundation for Basic Research under Grant No. 05-02-16914-a.

\section{APPENDIX A: ANGULAR REDUCTION}

\section{Designations and definitions}

The Coulomb interaction $g_{m n a b}$ is decomposed into the product of a term $J_{k}(m n a b)$

$$
\begin{aligned}
J_{k}(m m a b) & =\sum_{q}(-1)^{j_{m}-m_{m}+j_{n}-m_{n}+k-q} \times \\
& \left(\begin{array}{ccc}
j_{m} & k & j_{a} \\
-m_{m} & -q & m_{a}
\end{array}\right)\left(\begin{array}{ccc}
j_{n} & k & j_{b} \\
-m_{n} & q & m_{b}
\end{array}\right),
\end{aligned}
$$

depending on only the angular momentum quantum numbers $j_{i}$ and $m_{i}$ of the four states $(m, n, a, b)$, and a term $X_{k}($ mnab $)$ depending only on the principal quantum numbers $n_{i}$ and angular quantum numbers $\kappa_{i}$ of the states:

$$
g_{m n a b}=\sum_{k} J_{k}(m n a b) X_{k}(m n a b)
$$

Here

$X_{k}(m n a b)=(-1)^{k}\left\langle\kappa_{m}\left\|C^{k}\right\| \kappa_{a}\right\rangle\left\langle\kappa_{n}\left\|C^{k}\right\| \kappa_{b}\right\rangle R_{k}($ mnab $)$.

The quantities $R_{k}($ mnab) are (relativistic) Slater integrals and $\left\langle\kappa_{m}\left\|C^{k}\right\| \kappa_{a}\right\rangle$ is a reduced matrix elements of a normalized spherical harmonic. The quantities $Z_{k}(m n a b)$ are given by

$Z_{k}(m n a b)=X_{k}(m n a b)+\sum_{k^{\prime}}[k]\left\{\begin{array}{ccc}j_{m} & j_{a} & k \\ j_{n} & j_{b} & k^{\prime}\end{array}\right\} X_{k^{\prime}}(m n b a)$, 
TABLE V: Contributions of non-linear terms to the magnetic-dipole hyperfine constants $\mathrm{A}(\mathrm{MHz})$ of $\mathrm{Li}, \mathrm{Na}, \mathrm{K}, \mathrm{Rb}$, and $\mathrm{Cs}$ and comparison with experimental results. The experimental values are from Ref. [36] unless noted otherwise.

\begin{tabular}{|c|c|c|c|}
\hline $\mathrm{Li}$ & $2 s_{1 / 2}$ & $2 p_{1 / 2}$ & $2 p_{3 / 2}$ \\
\hline Core NL terms & -0.025 & -0.005 & 0.006 \\
\hline$S_{1 c} S_{1 v},\left\{S_{1 v} S_{2 c}, S_{1 c} S_{2 v}\right\}$ & -0.183 & -0.002 & -0.011 \\
\hline Other valence NL SD terms & 0.000 & 0.000 & 0.000 \\
\hline Total & 393.585 & 44.957 & -2.129 \\
\hline $\mathrm{SD}$ & 888.286 & 95.050 & 18.854 \\
\hline Core NL terms & -0.663 & -0.242 & -0.057 \\
\hline$S_{2 c} S_{2 v}$ & -2.645 & -1.022 & -0.193 \\
\hline$S_{1 c} S_{1 v},\left\{S_{1 v} S_{2 c}, S_{1 c} S_{2 v}\right\}$ & -4.878 & -0.696 & -0.174 \\
\hline Other valence NL SD terms & 0.001 & 0.000 & 0.001 \\
\hline $\mathrm{SD}$ & 237.159 & 28.696 & 6.214 \\
\hline Core NL terms & 0.457 & 0.138 & 0.032 \\
\hline$S_{2 c} S_{2 v}$ & -2.783 & -0.798 & -0.123 \\
\hline$S_{1 c} S_{1 v},\left\{S_{1 v} S_{2 c}, S_{1 c} S_{2 v}\right\}$ & -3.208 & -0.489 & -0.145 \\
\hline Other valence NL SD terms & 0.007 & 0.002 & 0.001 \\
\hline Total & 231.632 & 27.549 & 5.979 \\
\hline Expt. & 230.85 & $28.85(30)$ & $6.09(4)$ \\
\hline $\mathrm{Rb}$ & $5 s_{1 / 2}$ & $5 s_{1 / 2}$ & $5 p_{3 / 2}$ \\
\hline $\mathrm{SD}$ & 1051.554 & 125.624 & 25.560 \\
\hline Core NL terms & 5.319 & 0.710 & 0.155 \\
\hline$S_{2 c} S_{2 v}$ & -14.198 & -4.091 & -0.577 \\
\hline$S_{2 c} S_{2 v}$ & -39.441 & -12.871 & -1.137 \\
\hline$S_{1 c} S_{1 v},\left\{S_{1 v} S_{2 c}, S_{1 c} S_{2 v}\right\}$ & -80.300 & -11.259 & -2.489 \\
\hline Other valence NL SD terms & 0.321 & 0.043 & 0.015 \\
\hline Total & 2340.088 & 289.655 & 48.764 \\
\hline Expt. & 2298.2 & $291.89(8)^{(f)}$ & $50.275(3)^{(g)}$ \\
\hline
\end{tabular}
(a) Reference 37]
(b) Reference 38]
(c) Reference 39]
(d) Reference 40]
(e) Reference 41]
(f) Reference 42]
( $g$ ) Reference [3]

where $[k]=2 k+1$. Double excitation coefficients have the same angular structure as Coulomb matrix elements:

$$
\rho_{m n a b}=\sum_{k} J_{k}(m n a b) S_{k}(m n a b)
$$

and the quantities $\tilde{S}_{k}($ mnab) are defined in the same way as $Z_{k}($ mnab). The angular reductions for the singleexcitation coefficients are defined as follows: 


$$
\begin{gathered}
\rho_{m a}=\delta_{\kappa_{m} \kappa_{a}} \delta_{m_{m} m_{a}} S(m a), \\
\rho_{m v}=\delta_{\kappa_{m} \kappa_{v}} \delta_{m_{m} m_{v}} S(m v),
\end{gathered}
$$

where $\kappa$ is the relativistic angular momentum quantum number defined as

$$
\kappa=\mp(j+1 / 2) \text { for } j=l \pm 1 / 2 \text {. }
$$

2. Angular decomposition of terms contributing to the equation for core single excitation coefficients

$$
\begin{aligned}
G T_{1}^{s} & =\sum_{d r s} \sqrt{\frac{\left[j_{d}\right]}{\left[j_{a}\right]}} Z_{0}(m d r s) S(r a) S(s d) \\
& -\sum_{c d s} \sqrt{\frac{\left[j_{d}\right]}{\left[j_{a}\right]}} Z_{0}(c d a s) S(m c) S(s d) \\
G T_{1}^{s} & =-\sum_{c d r s l} \frac{(-1)^{r+s-a-d}}{[l]\left[j_{a}\right]} Z_{l}(c d s r) S_{l}(r s d a) S(m c) \\
& -\sum_{c d r s l} \frac{(-1)^{c+s-a-d}}{[l]\left[j_{a}\right]} Z_{l}(c d s r) S_{l}(s m c d) S(r a) \\
& +\sum_{c d r s} \sqrt{\frac{\left[j_{d}\right]}{\left[j_{a}\right]}} \delta_{j_{r} j_{c}} Z_{0}(c d r s) \tilde{S}_{0}(r m c a) S(s d) \\
G T_{3}^{s} & =-\sum_{c d r s} \sqrt{\frac{\left[j_{d}\right]}{\left[j_{a}\right]}} Z_{0}(c d s r) S(m c) S(r d) S(s a)
\end{aligned}
$$

3. Angular decomposition of the terms contributing to the equation for core double excitation coefficients

$$
\begin{aligned}
G T_{1}^{d} & =\sum_{r s} X_{l}(\text { mnrs }) S(r a) S(s b) \\
& +\sum_{c d} X_{l}(c d a b) S(m c) S(n d) \\
& -\left[\sum_{d r} Z_{l}(\text { mdar }) S(r b) S(n d)+\left(\begin{array}{c}
a \leftrightarrow b \\
m \leftrightarrow n
\end{array}\right)\right]
\end{aligned}
$$

$$
\begin{aligned}
& G T_{2}^{d}=\sum_{c d r} \frac{(-1)^{c+r+l}}{[l]} Z_{l}(c d r b) \tilde{S}_{l}(r m c a) S(n d) \\
& -\sum_{c d r} \sqrt{\frac{\left[j_{r}\right]}{\left[j_{a}\right]}} \delta_{j_{c} j_{a}} Z_{0}(c d a r) S_{l}(m n c b) S(r d) \\
& +\sum_{c d r j k}(-1)^{n+m+a+b}[l]\left\{\begin{array}{lll}
k & l & j \\
a & d & m
\end{array}\right\}\left\{\begin{array}{lll}
k & l & j \\
b & c & n
\end{array}\right\} \\
& \times \quad X_{j}(c d r a) S_{k}(n m c d) S(r b) \\
& -\sum_{c r s} \frac{(-1)^{l+c+s}}{[l]} Z_{l}(n c r s) \tilde{S}_{l}(\text { smca }) S(r b) \\
& +\sum_{c r s} \sqrt{\frac{\left[j_{c}\right]}{\left[j_{n}\right]}} \delta_{j_{r} j_{n}} Z_{0}(n c r s) S_{l}(m r a b) S(s c) \\
& -\sum_{c r s j k}(-1)^{n+m+a+b}[l]\left\{\begin{array}{lll}
k & l & j \\
n & s & b
\end{array}\right\}\left\{\begin{array}{ccc}
k & l & j \\
m & r & a
\end{array}\right\} \\
& \times X_{j}(n c r s) S_{k}(\operatorname{srab}) S(m c)+\left(\begin{array}{c}
a \leftrightarrow b \\
m \leftrightarrow n
\end{array}\right) \\
& G T_{3}^{d}=\sum_{c d r} X_{l}(c d a r) S(n d) S(m c) S(r b) \\
& -\sum_{c r s} X_{l}(m c r s) S(n c) S(r a) S(s b)+\left(\begin{array}{c}
a \leftrightarrow b \\
m \leftrightarrow n
\end{array}\right)
\end{aligned}
$$

$$
\begin{aligned}
G T_{4}^{d} & =\sum_{c d t u} \sum_{k_{1} k_{2} k_{3} k}(-1)^{a+b+t+u}[l][k] \\
& \times\left\{\begin{array}{ccc}
k_{1} & k_{3} & k \\
m & t & c
\end{array}\right\}\left\{\begin{array}{ccc}
k_{1} & k_{3} & k \\
n & u & d
\end{array}\right\}\left\{\begin{array}{ccc}
k_{2} & k & l \\
m & a & t
\end{array}\right\} \\
& \times\left\{\begin{array}{ccc}
k_{2} & k & l \\
n & b & u
\end{array}\right\} X_{k_{1}}(c d t u) S_{k_{2}}(\text { tuab }) S_{k_{3}}(\text { mncd }) \\
& +\sum_{c d t u} \frac{(-1)^{c+d+t+u}}{[l]^{2}} Z_{l}(c d t u) \tilde{S}_{l}(\text { mtac }) \tilde{S}_{l}(\text { undb) }) \\
& -\left[\sum_{c d t u k} \frac{(-1)^{c+d+t+u}}{[l]\left[j_{b}\right]} \delta_{j_{b} j_{c}} Z_{k}(c d t u) S_{k}(t u b d) S_{l}(\text { mnac })\right. \\
+ & \sum_{c d t u k} \frac{(-1)^{c+d+t+u}}{[l]\left[j_{m}\right]} \delta_{j_{t} j_{m}} Z_{k}(c d t u) S_{k}(\text { mucd }) S_{l}(n t b a) \\
& \left.+\left(\begin{array}{c}
a \leftrightarrow b \\
m \leftrightarrow n
\end{array}\right)\right]
\end{aligned}
$$




$$
\begin{aligned}
G T_{5}^{d} & =\sum_{c d t u j k}(-1)^{n+m+a+b}[l]\left\{\begin{array}{lll}
k & l & j \\
b & d & n
\end{array}\right\}\left\{\begin{array}{lll}
k & l & j \\
a & c & m
\end{array}\right\} \\
& \times X_{j}(c d t u) S_{k}(m n c d) S(t a) S(u b) \\
& +\sum_{c d t u j k}(-1)^{n+m+a+b}[l]\left\{\begin{array}{lll}
k & l & j \\
n & u & b
\end{array}\right\}\left\{\begin{array}{lll}
k & l & j \\
m & t & a
\end{array}\right\} \\
& \times X_{j}(c d t u) S_{k}(t u a b) S(m c) S(n d) \\
& -\left[\sum_{c d t u} \sqrt{\frac{\left[j_{c}\right]}{\left[j_{b}\right]}} \delta_{j_{d} j_{b}} Z_{0}(c d u t) S_{l}(\text { mnad }) S(t b) S(u c)\right. \\
& +\sum_{c d t u} \sqrt{\frac{\left[j_{c}\right]}{\left[j_{n}\right]}} \delta_{j_{n} j_{u}} Z_{0}(c d t u) S_{l}(\text { muab) } S(t c) S(n d) \\
& +\sum_{c d t u} \frac{(-1)^{u-d+l}}{[l]} Z_{l}(c d t u) \tilde{S}_{l}(\text { muad }) S(t b) S(n c) \\
& \left.+\left(\begin{array}{c}
a \leftrightarrow b \\
m \leftrightarrow n
\end{array}\right)\right] \\
& G T_{6}^{d}=\sum_{c d t u} X_{l}(c d t u) S(t a) S(u b) S(m c) S(n d)
\end{aligned}
$$

$$
\begin{aligned}
\delta E_{v}^{N L}= & -\sum_{c d t} \sqrt{\frac{\left[j_{d}\right]}{\left[j_{v}\right]}} Z_{0}(c d v t) S(t d) S(v c) \\
& +\sum_{d t u} \sqrt{\frac{\left[j_{d}\right]}{\left[j_{v}\right]}} Z_{0}(v d t u) S(t v) S(u d) \\
& -\sum_{c d u t k} \frac{1}{[k]\left[j_{v}\right]} Z_{k}(c d u t) S_{k}(u t v d) S(v c) \\
& -\sum_{c d u t k} \frac{1}{[k]\left[j_{v}\right]} Z_{k}(c d u t) S_{k}(u v c d) S(t v) \\
& +\sum_{c d t u} \sqrt{\frac{\left[j_{d}\right]}{\left[j_{v}\right]}} Z_{0}(c d t u) S_{0}(v t v c) S(u d) \\
& +\sum_{c d t u} \sqrt{\frac{\left[j_{d}\right]}{\left[j_{v}\right]}} Z_{0}(c d u t) S(t d) S(u v) S(v c)
\end{aligned}
$$

\section{Angular decomposition of terms contributing to} the core and valence energies

$$
\delta E_{c}^{N L}=\frac{1}{2} \sqrt{\left[j_{a}\right]\left[j_{b}\right]} Z_{0}(a b m n) S(m a) S(n b)
$$

[1] S. A. Blundell, W. R. Johnson, and J. Sapirstein, Phys. Rev. 43 43, 3407 (1991).

[2] M. S. Safronova, W. R. Johnson, and A. Derevianko, Phys. Rev. A 60, 4476 (1999).

[3] M. S. Safronova, A. Derevianko, and W. R. Johnson, Phys. Rev. A 58, 1016 (1998).

[4] M. S. Safronova, C. J. Williams, and C. W. Clark, Phys. Rev. A 69, 022509 (2004).

[5] M. S. Safronova and W. R. Johnson, Phys. Rev. A 62, 022112 (2000).

[6] A. Derevianko, W. R. Johnson, M. S. Safronova, and J. F. Babb, Phys. Rev. Lett. 82, 3589 (1999).

[7] F. Coester, Nucl. Phys. 7, 421 (1958).

[8] F. Coester and H. Kümmel, Nucl. Phys. 17, 477 (1960).

[9] J. Čižek, J. Chem. Phys. 45, 4256 (1966).

[10] J. Čižek, Adv. Chem. Phys. 14, 35 (1969).

[11] J. Čižek and J. Paldus, Int. J. Quantum Chem. 5, 359 (1971).

[12] T. D. Crawford and H. F. Schaefer III, Rev. Comp. Chem. 14, 33 (2002).

[13] A. Ynnerman, J. James, I. Lindgren, H. Persson, and S. Salomonson, Phys. Rev. A 50, 4671 (1994).

[14] E. Eliav, U. Kaldor, and Y. Ishikawa, Phys. Rev. A 50, 1121 (1994).
[15] I. S. Lim, M. Pernpointner, M. Seth, J. K. Laerdahl, P. Schwerdtfeger, P. Neogrady, and M. Urban, Phys. Rev. A 60, 2822 (1999).

[16] A.-M. Mårtensson-Pendrill, Mol. Phys. 98, 1201 (2000).

[17] A. Landau, E. Eliav, Y. Ishikawa, and U. Kaldor, J. Chem. Phys. 114, 2977 (2001).

[18] R. K. Chaudhuri, B. K. Sahoo, B. P. Das, H. Merlitz, U. S. Mahapatra, and D. Mukherjee, J. Chem. Phys. 119, 10633 (2003).

[19] S. G. Porsev and A. Derevianko, Phys. Rev. A 73, 12501 (2006).

[20] A. Derevianko and S. G. Porsev, submitted to Eur. J. Phys. A (2006) (hep-ph/0608178).

[21] B. K. Sahoo, R. Chaudhuri, B. P. Das, and D. Mukherjee, Phys. Rev. Lett. 96, 163003 (2006).

[22] I. Lindgren, Phys. Rev. A 31, 1273 (1985).

[23] S. Salomonson and A. Ynnerman, Phys. Rev. A 43, 88 (1991).

[24] S. A. Blundell, W. R. Johnson, Z. W. Liu, and J. Sapirstein, Phys. Rev. A 40, 2233 (1989).

[25] J. M. Grossman, R. P. Fliller III, T. E. Mehlstaubler, L. A. Orozco, M. R. Pearson, G. D. Sprouse, and W. Z. Zhao, Phys. Rev. A 62, 052507 (2000).

[26] M. Auzinsh, K. Bluss, R. Ferber, F. Gahbauer, A. Jar- 
mola, M. S. Safronova, U. I. Safronova, and M. Tamanis, Phys. Rev. A 75, 022502 (2007).

[27] C. S. Wood, S. C. Bennett, D. Cho, B. P. Masterson, J. L. Roberts, C. E. Tanner, and C. E. Wieman, Science 275, 1759 (1997).

[28] V. V. Flambaum and J. S. M. Ginges, Phys. Rev. A 72, 052115 (2005).

[29] G. E. Brown and D. G. Ravenhall, Proc. Roy. Soc. A 208, 552 (1951).

[30] W. R. Johnson, S. A. Blundell, and J. Sapirstein, Phys. Rev. A 37, 307 (1988).

[31] A. Derevianko and S. G. Porsev, Phys. Rev. A 71, 032509 (2005).

[32] C. E. Moore, Atomic Energy Levels, vol. 35 of Natl. Bur. Stand. Ref. Data Ser. (U.S. GPO, Washington, D.C., 1971).

[33] U. Volz and H. Schmoranzer, Phys. Scr. T65, 48 (1996).

[34] R. J. Rafac, C. E. Tanner, A. E. Livingston, and H. G.
Berry, Phys. Rev. A 60, 3648 (1999).

[35] P. Ragavan, At. Data Nucl. Data Tables 42, 189 (1989).

[36] W. Happer, Atomic Physics 4 (Plenum Press, New York, 1974).

[37] R. G. Schlecht and D. W. McColm, Phys. Rev. 142, 11 (1966).

[38] G. J. Ritter, Can. J. Phys. 43, 770 (1965).

[39] J. D. Lyons and R. K. Nesbet, Phys. Rev. Lett. 24, 433 (1970).

[40] W. A. Wijngaarden and J. Li, Z. Phys. D 32, 67 (1994).

[41] W. Yei, A. Sieradzan, and M. D. Havey, Phys. Rev. A 48, 1909 (1993).

[42] R. J. Rafac and C. E. Tanner, Phys. Rev. A 56, 1027 (1997).

[43] C. E. Tanner and C. Wieman, Phys. Rev. A 38, 1616 (1988). 